\title{
Comparison Theorems for Symmetric Functions of Characteristic Roots ${ }^{1}$
}

\section{Marvin Marcus}

\author{
(February 10, 1961)
}

\begin{abstract}
Several theorems are proved that give necessary and sufficient conditions for $A-B$ to be positive semidefinite Hermitian. The conditions are in terms of the comparison of elementary symmetric functions of the characteristic roots of $A+X$ and $B+X$ as $X$ varies over positive definite Hermitian matrices.
\end{abstract}

\section{Introduction}

In a recent paper $[4]^{2} \mathrm{M}$. Stone obtained the following result in reproving certain theorems of Ehrenfeld [1]: if $F$ and $G$ are positive definite $n$-square Hermitian matrices, then

$$
d(I+A F) \geq d(I+A G)
$$

for all positive definte n-square Hermitian matrices $A$ if and only if $F-G$ is positive semidefinite Hermitian. Here $d$ denotes determinant and henceforth $A>0$ $(\geq 0)$ will signify that $A$ is positive (positive semidefinite) Hermitian. $F \geq G(F>G)$ will mean $F-G$ $\geq 0(F-G>0)$. Note that since $A>0$ if and only if $\bar{A}^{-1}>0$ the condition (1) is the same as saying:

$$
d(A+F) \geq d(A+G)
$$

for all $A>0$. It is in the form (2) that we investigate what happens when we replace $d$ by some other elementary symmetric function of the characteristic roots. To be specific, suppose $E_{r}\left(x_{1}, \ldots, x_{n}\right)$ denotes the $r^{\text {th }}$ elementary symmetric function of the indicated variables:

$$
E_{r}\left(x_{1}, \cdots, x_{n}\right)=\sum_{1 \leq i_{1}<\ldots<i_{r} \leq n} \prod_{t=1}^{r} x_{i_{t}} .
$$

For a fixed $r, 1 \leq r \leq n$, let $E_{r}(A)$ denote $E_{r}\left(\lambda_{1}(A)\right.$, . . . , $\lambda_{n}(A)$ ) where $\lambda_{i}(A), i=1, \ldots, n$, are the characteristic roots of $A$. It the $\lambda_{i}(A)$ are real we will choose our notation so that $\lambda_{1}(A) \geq \ldots$. $\geq$ $\lambda_{n}(A)$. Then the problem we pose is to find conditions on the characteristic roots of $F$ and $G$ such that

$$
E_{r}(A+F) \geq E_{r}(A+G) \text { for all } A \geq 0 .
$$

We have

Theorem 1. Assume $F \geq 0, G \geq 0$, and $1 \leq r \leq n$.

1 This research was supported in part by the Office of Naval Research.
2 Figures in brackets indicate the literature references at the end for this paper.
If

$E_{r-p}\left(\lambda_{n}(F), \ldots, \lambda_{p+1}(F)\right) \geq E_{r-p}\left(\lambda_{1}(G), \ldots, \lambda_{n-p}(G)\right)$,

$p=0, \ldots, r-1$

it follows that

$$
E_{r}(A+F) \geq E_{r}(A+G) \text { for all } A \geq 0 .
$$

In case $\mathrm{G}=I$, (3) becomes

$$
E_{r-p}\left(\lambda_{n}(F), \cdots, \lambda_{p+1}(F)\right) \geq\left(\begin{array}{l}
n-p \\
r-p
\end{array}\right), \quad p=0, \cdots, r-1,
$$

which is both necessary and sufficient for

$$
E_{r}(A+F) \geq E_{r}(A+I) \text { for all } A \geq 0 .
$$

We remark that for $r=n$ the second part of theorem 1 simply becomes: $\lambda_{n}(F) \geq 1$ if and only if $d(A+F) \geq d(A+I)$ for all $A \geq 0$. Now in (1) multiply both sides by $d\left(A^{-1} R^{*} R\right)$ where $R$ is a nonsingular matrix satisfying $R^{*} G R=I, \quad R^{*} F R=K$ and we find that

$$
d\left(R^{*} A^{-1} R+K\right) \geq d\left(R^{*} A^{-1} R+I\right) .
$$

Now as $A$ runs over all positive definite matrices so does $R^{*} A^{-1} R$ and from the above remark we conclude that $\lambda_{n}(K) \geq 1$. The characteristic roots of $K$ are just the characeristic roots of $G^{-1} F$ and thus

$$
G^{-1 / 2} F G^{-1 / 2} \geq I, \quad F \geq G,
$$

and the result in [4] follows. A somewhat stronger multiplicative analogue of theorem 1 is available.

Theorem 2. Assume $F \geq 0, \quad G \geq 0$, and $1 \leq r \leq n$. Then

$$
E_{r}(A F) \geq E_{r}(A G) \text { for all } A \geq 0
$$


if and only if $\quad \prod_{j=1}^{r} \lambda_{j}\left(F^{-1} G\right) \leq 1$.

(8)

In order to prove these results we use some results concerning compound matrices and Grassmann products [2]. We give the coordinate definition of these items and list several of their properties. If $x_{1}$, . . ., $x_{r}$ are vectors in the unitary space of $n$-tuples, $x_{i}=\left(x_{i 1}, \ldots, x_{i n}\right), \quad 1 \leq r \leq n$, then $x_{1} \wedge \ldots \wedge x_{r}$ is the $\left(\begin{array}{l}n \\ r\end{array}\right)$-tuple whose coordinates are the r-square subdeterminants of the $\mathrm{r} \times n$ matrix $\left(x_{i j}\right), i=1, \ldots, r$, $j=1, \ldots, n$ arranged in lexicographic order. If $A$ is an $n$-square matrix, then $C_{r}(A)$ is the $\left(\begin{array}{l}n \\ r\end{array}\right)$-square matrix whose entries are the $r$-square subdeterminants of $A$ arranged in doubly lexicographic order according to the row and column indices of $A$. That is, if $1 \leq i_{1}<\ldots<i_{r} \leq n$ and $1 \leq j_{1}<\ldots<j_{r} \leq n$ are two increasing sets of $r$ integers then the $\left(i_{1}\right.$, ..., $\left.i_{r}\right),\left(j_{1}, \ldots, j_{r}\right)$ element of $C_{r}(A)$ is the determinant of the matrix $A\left[i_{1}, \ldots, i_{r} \mid j_{1}, \ldots . j_{r}\right]$ whose $(s, t)$ entry is $a_{i_{s} j_{t}}$. If $\dot{i}_{s}=j_{s}, s=1, \ldots, r$ we denote the corresponding principal submatrix by $A\left[i_{1}, \ldots\right.$, $i_{r}$. If $A \geq 0$ then $C_{r}(A) \geq 0 ; C_{r}(A) x_{1} \wedge \ldots \wedge x_{r}=A x \wedge$ $\ldots \wedge A x_{r}$; if $($,$) is the usual unitary inner product in$ the space of $m$-tuples then $\left(x_{1} \wedge \ldots \wedge x_{r}, y_{1} \wedge \ldots \wedge y_{r}\right)$ $=d\left\{\left(x_{i}, y_{j}\right)\right\}, \quad i, j=1, \ldots, r$.

We remark that the condition (3) of theorem 1 will not imply that $F \geq G$ in the case $r<n$. For even if $G=I$, we can easily construct $F$ so that $\lambda_{n}(F)<1$ and yet (3) holds for $p=0, \ldots, r-1$. However, there is a direct generalization of Stone's result to the compound matrix.

Theorem 3. If $F>0, \quad G>0$ and $1 \leq r \leq n$, then $C_{r}(I+A F) \geq C_{r}(I+A G)$ for all $A>0$

$$
\text { if and only if } F \geq G \text {. }
$$

Once again, $r=n$ is precisely the result in [4].

2. Proofs

We prove theorem 1. Let $A=U X U^{*}, U$ unitary, $X=\operatorname{diag}\left(x_{1}, \ldots, x_{n}\right)$. Then $E_{r}(A+F)-E_{r}(A+G)$ $=E_{r}\left(U^{*} A U+U^{*} F U\right)-E_{r}\left(U^{*} A U+U^{*} G U\right)=E_{r}(X$ $+K)-E_{r}(X+H)$ where $K=U^{*} F U \geq 0, H=U^{*} G U$ $\geq 0$. Let

$$
\varphi\left(x_{1}, \ldots, x_{n}: U\right)=E_{r}(X+K)-E_{r}(X+H) .
$$

Then

$$
\begin{aligned}
\varphi\left(x_{1}, \ldots, x_{n}: U\right) & =\sum_{1 \leq \alpha_{1}<\ldots<\alpha_{r} \leq n}\left\{d \left[\operatorname{diag}\left(x_{\alpha_{1}}, \ldots, x_{\alpha_{r}}\right)\right.\right. \\
& \left.+K\left[\alpha_{1}, \ldots, \alpha_{r}\right]\right]-d\left[\operatorname{diag}\left(x_{\alpha_{1}}, \ldots, x_{\alpha_{r}}\right)\right. \\
& \left.\left.+H\left[\alpha_{1}, \ldots, \alpha_{r}\right]\right]\right\} .
\end{aligned}
$$

We see from (10) that $\varphi$ is a polynomial in $x_{1}$, . ., $x_{n}$ of degree $r$ in which the nonconstant terms form a multilinear polynomial. Let

$$
\varphi_{i_{1}, \ldots i_{p}}\left(x_{1}, \ldots, x_{n}: U\right)=\frac{\partial^{p} \varphi}{\partial x_{i_{1}} \ldots \partial x_{i_{p}}}\left(x_{1}, \ldots, x_{n}: U\right)
$$

and

$$
\varphi_{i_{1} \ldots i_{p}}^{\circ}=\varphi_{i_{1} \ldots i_{p}}(0, \ldots, 0: U) .
$$

Then

$$
\varphi\left(x_{1}, \ldots, x_{n}: U\right)=\sum_{p=0}^{r} \sum_{1 \leq i_{1}<\ldots<i_{p} \leq n} x_{i_{1}} \ldots x_{i_{p}} \varphi_{i_{1}}^{\circ} \ldots i_{p},
$$

where the term corresponding to $p=0$ is just $\varphi(0$, $\therefore ., 0: U)$. Let $w$ denote the increasing sequence $i_{1}<\ldots<i_{p}$. Unless $w$ is a subset of $\alpha_{1}<\ldots<\alpha_{r}$ the partial derivative satisfies

$$
\begin{gathered}
\frac{\partial^{p}}{\partial x_{i_{p}} \ldots \partial x_{j_{p}}}\left\{d\left[\operatorname{diag}\left(x_{\alpha_{1}}, \ldots, x_{\alpha_{r}}\right)+K\left[\alpha_{1} \ldots \alpha_{r}\right]\right]\right. \\
\left.-d\left[\operatorname{diag}\left(x_{\alpha_{1}}, \ldots, x_{\alpha_{r}}\right)+H\left[\alpha_{1}, \ldots, \alpha_{r}\right]\right]\right\}=0 .
\end{gathered}
$$

If $w$ is a subset of $\alpha_{1}<\ldots<\alpha_{\tau}$ let $\alpha_{1}^{w}<\ldots<\alpha_{r-p}^{w}$ be the ordered complementary set of $w$ in $\alpha_{1}<\ldots<\alpha_{r}$. In this case the derivative in (12) has the value

$d\left[\operatorname{diag}\left(x_{\alpha_{1}^{w}}^{w}, \ldots, x_{\alpha_{r-p}^{w}}^{w}\right)+K\left[\alpha_{1}^{w}, \ldots, \alpha_{r-p}^{w}\right]\right]$

$$
-d\left[\operatorname{diag}\left(x_{\alpha_{1}^{w}}^{w}, \ldots, x_{\alpha_{r-p}^{w}}^{w}\right)+H\left[\alpha_{1}^{w}, \ldots, \alpha_{r-p}^{w}\right]\right] .
$$

Setting $x_{1}=\ldots=x_{n}=0$ in (13) we have

$$
\begin{aligned}
\phi_{i_{1} \ldots i_{p}}^{0}=\sum_{1 \leq \alpha_{1}<\ldots<\alpha_{r} \leq n}\{d & K\left[\alpha_{1}^{w}, \ldots, \alpha_{r-p}^{w}\right] \\
& \left.-d\left(H\left[\alpha_{1}^{w}, \ldots, \alpha_{r-p}^{w}\right]\right)\right\},
\end{aligned}
$$

where $\Sigma^{\prime}$ indicates that the summation is taken only over those $\alpha_{1}<\ldots<\alpha_{r}$ containing $w=\left(i_{1}<\ldots<i_{p}\right)$ as a subset. Returning to (11) we set $x_{i_{1}}=\ldots=x_{i_{p}}=t$ and all other $x_{j}=0$. Then $\phi(0, \ldots, t, \ldots, t, \ldots 0: U)=$ $t^{p} \phi_{i_{1}}^{0} \cdots i_{p}+L_{p-1}(t)$ where $L_{p-1}$ is a polynomial of degree at most $p-1$ in $t$. Since $\phi\left(x_{1}, \ldots, x_{n}: U\right) \geq 0$ we conclude, by letting $t$ increase, that $\phi_{i_{1}}^{0} \cdots i_{p} \geq 0$. Conversely, if $\phi_{i_{1}}^{0} \ldots i_{p} \geq 0$ and $\phi(0, \ldots, 0, U) \geq 0$, then $\phi\left(x_{1}, \ldots, x_{n}: U\right) \geq 0$ for all non-negative $x_{1}, \ldots, x_{n}$. Let $j_{i}<\ldots<j_{n-p}$ denote the set complementary to $i_{1}<\ldots<i_{p}$ in $1, \ldots, n$. Let $e_{j}, j=1, \ldots, n$, be the unit $n$-tuple with 1 in position $j, 0$ elsewhere. We may rewrite (14) as

$$
\begin{array}{r}
\phi_{i_{1} \ldots i_{p}}^{0}=\sum^{\prime}\left\{d K\left[\sigma_{1}, \ldots, \sigma_{r-p}\right]-d H\left[\sigma_{1}, \ldots, \sigma_{r-p}\right]\right\} \\
=\sum^{\prime}\left\{\left(C_{r-p}(K) e_{\sigma_{1}} \wedge \ldots \wedge e_{\sigma_{r-p}}, e_{\sigma_{1}} \wedge \ldots \wedge e_{\sigma_{r-p}}\right)\right. \\
\left.-\left(C_{r-p}(H) e_{\sigma_{1}} \wedge \ldots \wedge e_{\sigma_{r-p}}, e_{\sigma_{1}} \wedge \ldots \wedge \epsilon_{\sigma_{r-p}}\right)\right\} \\
=\sum^{\prime}\left(C_{r-p}(F) u_{\sigma_{1}} \wedge \ldots \wedge u_{\sigma_{r-p}}, u_{\sigma_{1}} \wedge \ldots \wedge u_{\sigma_{r-p}}\right) \\
-\sum^{\prime}\left(C_{r-p}(G) u_{\sigma_{1}} \wedge \ldots \wedge u_{\sigma_{r-p}},\right. \\
\left.u_{\sigma_{1}} \wedge \ldots \wedge u_{\sigma_{r-p}}\right), \quad(15)
\end{array}
$$


where $\sum$ ' indicates that the summation extends over precisely those increasing sequence $\sigma_{1}<\ldots<\sigma_{r-p}$ which are subsets of $j_{1}<\ldots<j_{n-p}$. Also $u_{j}=U e_{j}$, $j=1, \ldots, n$ is an orthonormal set of vectors (recall that $U$ is unitary). We use an extremal result in [3: theorem 1, p. 525] to conclude from (15) that

$$
\begin{aligned}
\phi_{i_{1} \ldots i_{p}}^{0} \geq E_{r-p}\left(\lambda_{p+1}(F)\right. & \left., \ldots, \lambda_{n}(F)\right) \\
& -E_{r-p}\left(\lambda_{1}(G), \ldots, \lambda_{n-p}(G)\right) \geq 0 .
\end{aligned}
$$

In case $G=I$ we have from (15) again that

$$
\begin{aligned}
\phi_{i_{1} \ldots i_{p}}^{0}=\sum^{\prime}\left(C_{r-p}(F) u_{\sigma_{1}} \wedge \ldots\right. & \wedge u_{\sigma_{r-p}}, \\
& \left.u_{\sigma_{1}} \wedge \ldots \wedge u_{\sigma_{r-p}}\right)-\left(\begin{array}{c}
n-p \\
r-p
\end{array}\right)
\end{aligned}
$$

for all choices of sets of $n-p$ orthonormal vectors $u_{1}, \ldots, u_{n-p}$, and another application of the above cited extremal result completes the proof.

To proceed to the proof of theorem 2, choose a nonsingular $R$ such that $F=R R^{*}, \quad G=R D R^{*}$, $D=\operatorname{diag}\left(\lambda_{1}\left(F^{-1} G\right)\right.$. . ., $\left.\lambda_{n}\left(F^{-1} G\right)\right)$. Let $P$ be an arbitrary nonsingular matrix and since any $A>0$ is of the form $\left(P R^{-1}\right) *\left(P R^{-1}\right)$ we have that $(7)$ is equivalent to

$$
\begin{aligned}
E_{r}\left(\left(P R^{-1}\right) *\left(P R^{-1}\right) R R^{*}\right) & \geq E_{r}\left(\left(P R^{-1}\right) *\left(P R^{-1}\right) R D R^{*}\right), \\
E_{r}\left(P^{*} P\right) & \geq E_{r}\left(P^{*} P D\right),
\end{aligned}
$$

or

$$
E_{r}(A) \geq E_{r}(A D) \text { for all } A>0 \text {. }
$$

In (16) replace $A$ by $V X V^{*}, V$ unitary, $X=$ diag $\left(x_{1}, \ldots, x_{n}\right) \geq 0$ to obtain

or

$$
E_{r}(X) \geq E_{r}(X H), \quad H=V^{*} D V
$$

$$
\operatorname{tr}\left[C_{r}(X)\left(I-C_{r}(H)\right)\right]=0,
$$

where $I$ is the $\left(\begin{array}{l}n \\ r\end{array}\right)$-square identity matrix. It is not difficult to check that (17) holds for all non-negative diagonal $X$ if and only if every diagonal element of $I-C_{r}(H)$ is non-negative. That is,

$$
1-\left(C_{r}\left(V^{*} D V\right) e_{\alpha_{1}} \wedge \ldots \wedge e_{\alpha_{r}}, \quad e_{\alpha_{1}} \wedge \ldots \wedge e_{\alpha_{r}}\right) \geq 0
$$

must hold for all $V$ and all $1 \leq \alpha_{1}<\ldots<\alpha_{r} \leq n$. But this is precisely equivalent to

$$
\left(C_{r}(D) u_{\alpha_{1}} \wedge \ldots \wedge u_{\alpha_{r}}, \quad u_{\alpha_{1}} \wedge \ldots \wedge u_{\alpha_{r}}\right) \leq 1
$$

for all orthonormal $u_{\alpha_{1}}, \ldots, u_{\alpha_{r}}$. As in the proof of theorem 1 we have finally that (7) holds if and only if (8) does.

To prove theorem 3 it will be convenient to let $\stackrel{c}{=}$ denote the relation of Hermitian congruence. Then if $A>0$,

$$
\begin{aligned}
& C_{r}(I+A F)-C_{r}(I+A G)=C_{r}(A) \\
& \left\{C_{r}\left(A^{-1}+F\right)-C_{r}\left(A^{-1}+G\right)\right\} \stackrel{c}{=}\left(C_{r}(A)\right)^{1 / 2} \\
& {\left[C_{r}\left(A^{-1}+F\right)-C_{r}\left(A^{-1}+G\right)\right]\left(C_{r}(A)\right)^{-1 / 2} .}
\end{aligned}
$$

This last matrix has the same roots as the Hermitian matrix $C_{r}\left(A^{-1}+F\right)-C_{r}\left(A^{-1}+G\right)$ and hence (9) is equivalent to

Now

$$
C_{r}(A+F) \geq C_{r}(A+G) \quad \text { for all } A>0 .
$$

$$
\begin{aligned}
& C_{r}(A+F)-C_{r}(A+G)=C_{r}(A+F)-\left(C_{r}(G)\right)^{1 / 2} \\
& \quad \begin{array}{l}
C_{r}\left(G^{-1 / 2} A G^{-1 / 2}+I\right)\left(C_{r}(G)\right)^{1 / 2} \stackrel{c}{=} C_{r}\left(G^{-1 / 2} A G^{-1 / 2}\right. \\
\left.\quad+G^{-1 / 2} F G^{-1 / 2}\right)-C_{r}\left(G^{-1 / 2} A G^{-1 / 2}+I\right) .
\end{array}
\end{aligned}
$$

Thus (9) is equivalent to

$$
C_{r}(A+H)-C_{r}(A+I) \geq 0 \text { for all } A>0
$$

where $H=G^{-1 / 2} H G^{-1 / 2}$.

By a unitary congruence we may assume $H=\operatorname{diag}$ $\left(h_{1}, \ldots, h_{n}\right)$ and by setting $A=\operatorname{diag}\left(x_{1}, \ldots, x_{n}\right) \geq 0$ we see that

$$
\prod_{t=1}^{r}\left(x_{i_{t}}+h_{i_{t}}\right) \geq \prod_{t=1}^{r}\left(x_{i_{t}}+1\right)
$$

for any non-negative numbers $x_{i_{1}}, \ldots, x_{i_{r}}$. This clearly implies that each $h_{t} \geq 1, \quad t=1, \ldots, n$. Thus, $0 \geq H-I=G^{-1 / 2} F G^{-1 / 2}-I \stackrel{c}{=} F-G$.

Conversely suppose $F-G>0$. Then $H>I$ and if we set $B=A+I>0$ we would like to conclude that

$C_{r}(A+H)=C_{r}(B+H-I) \geq C_{r}(B)=C_{r}(A+I)$.

But (19) is equivalent to

$$
C_{r}(I+K) \geq C_{r}(I), \quad K=B^{-1 / 2}(H-I) B^{-1 / 2} .
$$

After a unitary congruence (20) reduces to

$$
C_{r}\left(I+\operatorname{diag}\left(k_{1}, \ldots, k_{n}\right)\right) \geq C_{r}(I),
$$

where $k_{\alpha} \geq 0$ are the characteristic roots of $K$. The proof is complete.

\section{References}

[1] S. Ehrenfeld, Complete class theorems in experimental design, Proc. of the Third Berkeley Symposium on Mathematical Statistics and Probability 1, 57 (19541955), Univ. of Calif. Press, Berkeley, 1956; Math. Rev. 18, 946

[2] W. Graeub, Lineare Algebra, ch. 5 (Springer-Verlag Berlin, 1958).

[3] M. Marcus and J. L. McGregor, Extremal properties of Hermitian matrices. Can. J. Math. 8, 524 (1956).

[4] M. Stone,Application of a measure of information to the design and comparison of regression experiments. Ann. Math. Statist. 30, 55 (1959); Math. Rev. 21, 980

(Paper 65B2-49) 